\title{
Symmetric polynomials and symmetric mean inequalities
}

\author{
Karl Mahlburg * \\ Department of Mathematics \\ Louisiana State University \\ Baton Rouge, LA 70803, U.S.A. \\ mahlburg@math.lsu.edu
}

\author{
Clifford Smyth \\ Department of Mathematics and Statistics \\ University of North Carolina Greensboro \\ Greensboro, NC 27402, U.S.A. \\ cdsmyth@uncg.edu
}

Submitted: Nov 22, 2012; Accepted: Aug 27, 2013; Published: Sep 6, 2013

Mathematics Subject Classifications: 05E05, 26E60, 60C05

\begin{abstract}
We prove generalized arithmetic-geometric mean inequalities for quasi-means arising from symmetric polynomials. The inequalities are satisfied by all positive, homogeneous symmetric polynomials, as well as a certain family of nonhomogeneous polynomials; this family allows us to prove the following combinatorial result for marked square grids.

Suppose that the cells of a $n \times n$ checkerboard are each independently filled or empty, where the probability that a cell is filled depends only on its column. We prove that for any $0 \leqslant \ell \leqslant n$, the probability that each column has at most $\ell$ filled sites is less than or equal to the probability that each row has at most $\ell$ filled sites.
\end{abstract}

Keywords: symmetric means; symmetric polynomials; arithmetic-geometric mean inequality

\section{Introduction}

Let $n$ be a positive integer. We define an $n$-variable orthant function to be a continuous function $F: \mathbb{R}_{\geqslant 0}^{n} \rightarrow \mathbb{R}_{\geqslant 0}$ such that $F(\boldsymbol{x})=F\left(x_{1}, \ldots, x_{n}\right)$ is monotonically increasing in each $x_{i}$ and that also has a strictly increasing diagonal restriction, $f_{F}(y)=F(y, \ldots, y)$. Given an $n$-variable orthant function $F$, we define the following $n$-variable orthant functions associated with $F$ : the quasi-arithmetic mean, the quasi-geometric mean, and the

* Partially supported by an NSF Postdoctoral Fellowship administered by the Mathematical Sciences Research Institute through its core grant DMS-0441170 and by NSF Grant DMS-1201435.. 
quasi-mean; respectively, they are

$$
\begin{aligned}
\mathcal{A}_{F}(\boldsymbol{x}) & :=f_{F}^{-1}\left(\frac{f_{F}\left(x_{1}\right)+\cdots+f_{F}\left(x_{n}\right)}{n}\right), \\
\mathcal{G}_{F}(\boldsymbol{x}) & :=f_{F}^{-1}\left(\prod_{j=1}^{n} f_{F}\left(x_{j}\right)^{\frac{1}{n}}\right), \\
\mathcal{M}_{F}(\boldsymbol{x}) & :=f_{F}^{-1}(F(x)) .
\end{aligned}
$$

Note that these means have been studied classically (see [2], Chapter III).

Some care is needed to verify that these definitions are well-defined. One must note that since $f_{F}(y)$ is strictly increasing and continuous, its range $R:=f_{F}\left(\mathbb{R}_{\geqslant 0}\right)$ is of the form $R=\left[f_{F}(0), M\right)$ or $\left[f_{F}(0),+\infty\right)$, according to the value $M=\lim _{y \rightarrow+\infty} f_{F}(y)$. Furthermore, $f_{F}$ is a bijection and $f_{F}^{-1}$ is strictly increasing and continuous. Since $R$ is closed under taking arithmetic and geometric means of its elements, $\mathcal{A}_{F}(x)$ and $\mathcal{G}_{F}(x)$ are well-defined. Since $F$ is monotonically increasing in each variable, it also satisfies

$$
f_{F}(\underline{\boldsymbol{x}}) \leqslant F(\boldsymbol{x}) \leqslant f_{F}(\overline{\boldsymbol{x}})
$$

where $\underline{\boldsymbol{x}}:=\min \left\{x_{i}: 1 \leqslant i \leqslant n\right\}$ and $\overline{\boldsymbol{x}}:=\max \left\{x_{i}: 1 \leqslant i \leqslant n\right\}$. This implies that $F\left(\mathbb{R}_{\geqslant 0}^{n}\right)=R$ and so $\mathcal{M}_{F}(x)$ is well-defined also.

If $M=\mathcal{A}_{F}, \mathcal{G}_{F}$, or $\mathcal{M}_{F}$, we therefore have that $M$ is a quasi-mean. In particular, $M$ satisfies the following usual properties of a mean: it is continuous and monotonically increasing in each variable, $\underline{\boldsymbol{x}} \leqslant M(\boldsymbol{x}) \leqslant \overline{\boldsymbol{x}}$ for all $\boldsymbol{x} \in \mathbb{R}_{\geqslant 0}$, and $M(y, \ldots, y)=y$ for all $y \geqslant 0$. Note that the $f_{F}^{-1}$ in the definition of $M$ ensures the final "identity" property, $M(y, \ldots, y)=y$. However, $M$ is not necessarily linearly homogeneous, i.e. we do not necessarily have $M(\lambda \boldsymbol{x})=\lambda M(\boldsymbol{x})$ for all $\lambda \geqslant 0$ and $\boldsymbol{x} \in \mathbb{R}_{\geqslant 0}^{n}$. The function $F(\boldsymbol{x})=\left(1+x_{1}\right)\left(1+x_{2}\right)$ provides a simple counterexample for each type of $M$.

Since $f_{F}$ and hence $f_{F}^{-1}$ are strictly increasing, $\mathcal{A}_{F}$ and $\mathcal{G}_{F}$ are strictly increasing in each variable. An arithmetic-geometric mean inequality between $\mathcal{G}_{F}$ and $\mathcal{A}_{F}$ also easily follows: for all $\boldsymbol{x} \in \mathbb{R}_{\geqslant 0}^{n}$,

$$
\mathcal{G}_{F}(\boldsymbol{x}) \leqslant \mathcal{A}_{F}(\boldsymbol{x})
$$

with equality if and only if all $x_{i}$ are equal. This is also Theorem 85 of [2], with $\psi=\log f_{F}$, $\chi=f_{F}$, and $q \equiv 1 / n$. Note that the classical arithmetic-geometric mean inequality can be recovered from this by setting $F(\boldsymbol{x})=\left(x_{1} \cdots x_{n}\right)^{1 / n}$.

In this paper we study functions whose quasi-means provide a refinement of the preceding arithmetic-geometric mean inequality. Namely, we are interested in $\mathcal{S}$, which we define to be the set of all orthant functions $F$ for which

$$
\mathcal{G}_{F}(\boldsymbol{x}) \leqslant \mathcal{M}_{F}(x) \leqslant \mathcal{A}_{F}(\boldsymbol{x}), \quad \text { for all } \boldsymbol{x} \in \mathbb{R}_{\geqslant 0}^{n} \text {. }
$$

Proposition 1.1. The set $\mathcal{S}$ satisfies the following properties.

1. If $F\left(x_{1}, \ldots, x_{n}\right)$ is a homogeneous symmetric polynomial with positive coefficients, then $F \in \mathcal{S}$. 
2. If $F_{i}\left(x_{1}, \ldots, x_{n}\right) \in \mathcal{S}$ for $i=1,2$, then $F_{1} \cdot F_{2} \in \mathcal{S}$.

Remark 1.2. The set $\mathcal{S}$ is not closed under addition. For example, $F(\boldsymbol{x})=1+x_{1} x_{2}$ is the sum of two homogeneous functions but $\mathcal{G}_{F} \leqslant \mathcal{M}_{F}$ fails to hold when $x_{1} \neq x_{2}$.

For integers $\ell$ and $n$ with $0 \leqslant \ell \leqslant n$ we define

$$
\eta_{\ell}(\boldsymbol{x}):=\sum_{j=0}^{\ell} \sum_{\substack{I \subseteq[n] \\|I|=j}} x_{I},
$$

where we have adopted the common notation $x_{I}:=\prod_{i \in I} x_{i}$, along with the convention $x_{\varnothing}=1$. Note that $\eta_{\ell}$ is an orthant function if and only if $\ell \geqslant 1$. We denote the diagonal restriction of $\eta_{\ell}$ by

$$
\mu_{\ell}(y):=\eta_{\ell}(y, \ldots, y)=\sum_{j=0}^{\ell}\left(\begin{array}{l}
n \\
j
\end{array}\right) y^{j} .
$$

In Section 3 we will prove the following result, which states that $\eta_{\ell} \in \mathcal{S}$ for $1 \leqslant \ell \leqslant n$.

Theorem 1.3. If $\ell$ and $n$ are integers with $0 \leqslant \ell \leqslant n$ and $n \geqslant 1$, then

$$
\left(\prod_{i=1}^{n} \mu_{\ell}\left(x_{i}\right)\right)^{1 / n} \leqslant \eta_{\ell}(\boldsymbol{x}) \leqslant \frac{1}{n} \sum_{i=1}^{n} \mu_{\ell}\left(x_{i}\right), \text { for all } \boldsymbol{x} \in \mathbb{R}_{\geqslant 0}^{n}
$$

Equality holds throughout if and only if $\ell=0, \ell=n$, or $x_{1}=\cdots=x_{n}$.

These quasi-mean inequalities have an appealing application to combinatorial probability. Let $\left\{X_{i j}, 1 \leqslant i, j \leqslant n\right\}$ be a collection of independent Bernoulli random variables with probability $p_{j}$; in other words, $\mathbf{P}\left(X_{i j}=1\right)=p_{j}$ and $\mathbf{P}\left(X_{i j}=0\right)=1-p_{j}$. Using these, we further define the random variables

$$
\begin{aligned}
& C_{j}:=\sum_{i=1}^{n} X_{i j} \text { for } 1 \leqslant j \leqslant n, \\
& R_{i}:=\sum_{j=1}^{n} X_{i j} \quad \text { for } 1 \leqslant i \leqslant n .
\end{aligned}
$$

Using Theorem 1.3, we prove bounds relating the distributions of the $C_{j}$ s and $R_{i}$ s.

Theorem 1.4. Suppose that $p_{1}, \ldots, p_{n} \in[0,1]$. If $\ell$ is an integer with $0 \leqslant \ell \leqslant n$, then

$$
\mathbf{P}\left(\max _{1 \leqslant j \leqslant n}\left\{C_{j}\right\} \leqslant \ell\right) \leqslant \mathbf{P}\left(\max _{1 \leqslant i \leqslant n}\left\{R_{i}\right\} \leqslant \ell\right)
$$


Theorem 1.4 has two immediate combinatorial reformulations: one to marked square grids and another to random bipartite graphs. First, suppose that markers are independently placed in the squares of an $n$ by $n$ grid such that the probability that a marker is placed in a square depends only on that square's column. Then for all integers $\ell$ with $0 \leqslant \ell \leqslant n$, the probability that every column has at most $\ell$ markers is less than or equal to the probability that each row has at most $\ell$ markers.

Alternatively, suppose that $G$ is a finite complete bipartite graph with bipartition $(A, B)$, where $|A|=|B|=n$. Let $H$ be a random subgraph of $G$ where each edge $e$ of $G$ is independently selected to belong to $H$ with a probability that depends only on the left vertex $e \cap A$. If $\ell \geqslant 0$, then

$$
\mathbf{P}\left(\max _{a \in A}\left\{d_{H}(a)\right\} \leqslant \ell\right) \leqslant \mathbf{P}\left(\max _{b \in B}\left\{d_{H}(b)\right\} \leqslant \ell\right) .
$$

Remark 1.5. In the grid formulation, both events in the inequality require that at most $n \ell$ squares be occupied. Similarly, both events in the bipartite graph formulation require that there be at most $n \ell$ edges.

The remainder of the paper is as follows. In Section 2 we study the basic properties of homogeneous symmetric polynomials and the functions in $\mathcal{S}$, and also prove Proposition 1.1. In Section 3 we use Lagrange multipliers and polynomial inequalities to prove Theorem 1.3. We conclude in Section 4, where we describe the relationship between the quasi-mean inequalities and combinatorial probability, and prove Theorem 1.4.

\section{Properties of symmetric polynomials and $\mathcal{S}$}

As found in Chapter 7 of [4], one can define various homogeneous (graded) bases for the ring of symmetric polynomials on $n$ variables; we will make explicit use of the elementary symmetric polynomials $\left\{e_{j}(\boldsymbol{x}) \mid 0 \leqslant j \leqslant n\right\}$, where the degree $j$ polynomial is defined as $e_{j}(\boldsymbol{x}):=\sum\left\{x_{I}: I \subseteq[n],|I|=j\right\}$. We will also use the monomial symmetric polynomials, which are defined as follows.

For a positive integer $n$, let $\Delta(n):=\left\{\boldsymbol{\lambda} \in \mathbb{R}_{\geqslant 0}^{n}: \lambda_{1} \geqslant \cdots \geqslant \lambda_{n}\right\}$, and define the weight of such a vector $\boldsymbol{\lambda}$ as $|\boldsymbol{\lambda}|:=\lambda(1)+\cdots+\lambda(n)$. If $\boldsymbol{\lambda} \in \Delta(n)$, the monomial symmetric polynomial associated to $\boldsymbol{\lambda}$ is defined as

$$
M_{\boldsymbol{\lambda}}(\boldsymbol{x}):=\frac{1}{n !} \sum_{\sigma \in S_{n}} x_{\sigma(1)}^{\lambda(1)} \cdots x_{\sigma(n)}^{\lambda(n)} .
$$

Note that this is homogeneous of degree $|\boldsymbol{\lambda}|$.

We now recall various inequalities between symmetric polynomials. Suppose that $\boldsymbol{\lambda}_{\boldsymbol{i}}=\left(\lambda_{i}(1), \ldots, \lambda_{i}(n)\right) \in \Delta(n)$ for $i \in\{1,2\}$. We say that $\boldsymbol{\lambda}_{\mathbf{1}}$ majorizes $\boldsymbol{\lambda}_{\mathbf{2}}$ if and only if $\left|\boldsymbol{\lambda}_{\mathbf{1}}\right|=\left|\boldsymbol{\lambda}_{\mathbf{2}}\right|$, and

$$
\lambda_{1}(1)+\cdots+\lambda_{1}(j) \geqslant \lambda_{2}(1)+\cdots+\lambda_{2}(j)
$$

for all $1 \leqslant j<n$. In this case, we write $\boldsymbol{\lambda}_{\mathbf{1}} \succeq \boldsymbol{\lambda}_{\mathbf{2}}$. Muirhead's inequalities can be concisely stated as follows. 
Theorem (Muirhead [3]). Suppose that $\boldsymbol{\lambda}_{\mathbf{1}}, \boldsymbol{\lambda}_{\mathbf{2}} \in \Delta(n)$. The inequality

$$
M_{\boldsymbol{\lambda}_{\mathbf{1}}}(\boldsymbol{x}) \geqslant M_{\boldsymbol{\lambda}_{\mathbf{2}}}(\boldsymbol{x})
$$

is satisfied for all $\boldsymbol{x} \in \mathbb{R}_{\geqslant 0}^{n}$ if and only if $\boldsymbol{\lambda}_{\mathbf{1}} \succeq \boldsymbol{\lambda}_{\mathbf{2}}$.

Note that [3] only contains the case where both $\boldsymbol{\lambda}_{\boldsymbol{i}}$ have integral parts, while [2] (Theorem 45, p. 45) contains the general result above. We also recall the following elementary result from the general theory of series inequalities; see [2] (Theorem 368, p. 261).

Theorem (Rearrangement Inequality). Suppose that $a_{1} \geqslant \ldots \geqslant a_{n} \geqslant 0$ and $b_{1} \geqslant \ldots \geqslant$ $b_{n} \geqslant 0$. If $\sigma \in S_{n}$ is a permutation, then

$$
\sum_{i=1}^{n} a_{i} b_{i} \geqslant \sum_{i=1}^{n} a_{i} b_{\sigma(i)}
$$

Our final preliminary observations address the inequalities in (1.2) individually. Let $\mathcal{L}$ denote the set of orthant functions $F$ that satisfy the left inequality:

$$
\mathcal{G}_{F}(\boldsymbol{x}) \leqslant \mathcal{M}_{F}(\boldsymbol{x}), \text { for all } \boldsymbol{x} \in \mathbb{R}_{\geqslant 0}^{n},
$$

and let $\mathcal{R}$ denote the set of orthant functions that satisfy the right inequality:

$$
\mathcal{M}_{F}(\boldsymbol{x}) \leqslant \mathcal{A}_{F}(\boldsymbol{x}), \text { for all } \boldsymbol{x} \in \mathbb{R}_{\geqslant 0}^{n} .
$$

Clearly we have $\mathcal{S}=\mathcal{L} \cap \mathcal{R}$. The following properties follow immediately from the definitions of the quasi-means.

Proposition 2.1. The classes $\mathcal{L}$ and $\mathcal{R}$ have the following closure properties:

1. If $F_{i} \in \mathcal{L}$ for $i=1,2$, then $F_{1}^{a} \cdot F_{2}^{b} \in \mathcal{L}$ for all $a, b \geqslant 0$.

2. If $F_{i} \in \mathcal{R}$ for $i=1,2$, then $a F_{1}+b F_{2} \in \mathcal{R}$ for all $a, b \geqslant 0$.

The preceding facts now allow us to prove our first result about $\mathcal{S}$.

Proof of Proposition 1.1. We first prove statement 1. Suppose $F$ is a homogeneous symmetric polynomial with positive coefficients and total degree $w$. It can be written as

$$
F(\boldsymbol{x})=\sum_{i=1}^{k} a_{i} M_{\boldsymbol{\lambda}_{\boldsymbol{i}}}(\boldsymbol{x})
$$

where $a_{i}>0, \boldsymbol{\lambda}_{\boldsymbol{i}} \in \Delta(n)$ and $\left|\boldsymbol{\lambda}_{\boldsymbol{i}}\right|=w$ for all $i$. Writing $A:=\sum_{i=1}^{k} a_{i}>0$, Muirhead's theorem (2.2) then implies that

$$
A \cdot M_{(w / n, \ldots, w / n)}(\boldsymbol{x}) \leqslant F(\boldsymbol{x}) \leqslant A \cdot M_{(w, 0, \ldots, 0)}(\boldsymbol{x}), \text { for all } \boldsymbol{x} \in \mathbb{R}_{\geqslant 0}^{n} .
$$


We finish the proof by showing that this is equivalent to the statement $F \in \mathcal{S}$. Since $f_{F}(y)=F(y, \ldots, y)=A y^{w}$, we find that the leftmost expression in (2.5) is the same as

$$
A \cdot \frac{1}{n !} \cdot\left(x_{1} \cdots x_{n}\right)^{w / n} \cdot n !=\left(\prod_{i=1}^{n} f_{F}\left(x_{i}\right)\right)^{1 / n}
$$

Similarly, the rightmost expression in (2.5) equals

$$
A \cdot \frac{1}{n !}\left(x_{1}^{w}+\cdots+x_{n}^{w}\right) \cdot(n-1) !=\frac{1}{n} \sum_{i=1}^{n} f_{F}\left(x_{i}\right) \text {. }
$$

This completes the first part of the proof.

We now turn to statement 2. Throughout this part of the proof we write $f_{i}$ as shorthand for $f_{F_{i}}$. By part 1 of Proposition 2.1 we need only show that if $F_{i} \in \mathcal{S}$ for $i=1,2$, then $F_{1} \cdot F_{2} \in \mathcal{R}$. This is equivalent to showing that

$$
\left(F_{1} F_{2}\right)(\boldsymbol{x}) \leqslant \frac{1}{n} \sum_{i=1}^{n} f_{F_{1} F_{2}}(\boldsymbol{x}),
$$

and we can immediately rewrite $f_{F_{1} F_{2}}=f_{1} \cdot f_{2}$. Using the assumption that $F_{i} \in \mathcal{R}$, we find that the left side of (2.6) satisfies

$$
\left(F_{1} F_{2}\right)(\boldsymbol{x}) \leqslant\left(\frac{1}{n} \sum_{i=1}^{n} f_{1}\left(x_{i}\right)\right)\left(\frac{1}{n} \sum_{i=1}^{n} f_{2}\left(x_{i}\right)\right)=\frac{1}{n^{2}} \sum_{i_{1}, i_{2}=1}^{n} f_{1}\left(x_{i_{1}}\right) f_{2}\left(x_{i_{2}}\right) .
$$

Define the one-step shift cyclical permutation by $\sigma(i):=i+1$ for $1 \leqslant i \leqslant n-1$, and $\sigma(n):=1$. Reordering the $x_{i}$ if necessary so that $x_{1} \geqslant \ldots \geqslant x_{n} \geqslant 0$, we then further rewrite the sum from $(2.7)$ as

$$
\frac{1}{n^{2}} \sum_{i_{1}, i_{2}=1}^{n} f_{1}\left(x_{i_{1}}\right) f_{2}\left(x_{i_{2}}\right)=\frac{1}{n^{2}} \sum_{j=0}^{n-1} \sum_{i=1}^{n} f_{1}\left(x_{i}\right) f_{2}\left(x_{\sigma^{j}(i)}\right) \text {. }
$$

The Rearrangement Inequality, (2.3), now implies that the largest term in the outer summation occurs when $j=0$, so

$$
\left(F_{1} F_{2}\right)(\boldsymbol{x}) \leqslant \frac{1}{n} \sum_{i=1}^{n}\left(f_{1} f_{2}\right)\left(x_{i}\right)
$$

which verifies (2.6). 


\section{Proof of Theorem 1.3}

\subsection{Overview}

In this section we prove Theorem 1.3, devoting most of our effort to the left inequality. In particular, we consider the level sets of $\eta_{\ell}(\boldsymbol{x})$ and then apply the technique of Lagrange multipliers in order to determine the extremal behavior of

$$
U(\boldsymbol{x}):=\prod_{j=1}^{n} \mu_{\ell}\left(x_{j}\right)^{\frac{1}{n}}
$$

For each $R$ in the range of $\eta_{\ell}$ on $\mathbb{R}_{\geqslant 0}^{n}$, define the surface $\Omega(R)=\left\{\boldsymbol{x} \in \mathbb{R}_{\geqslant 0}^{n}: \eta_{\ell}(\boldsymbol{x})=R\right\}$. Given $d>0$ and an integer $k$ with $1 \leqslant k \leqslant n$, we define $\boldsymbol{c}_{k}(d)=d\left(\boldsymbol{e}_{\boldsymbol{1}}+\cdots+\boldsymbol{e}_{\boldsymbol{k}}\right) \in \mathbb{R}^{n}$, where $\boldsymbol{e}_{\boldsymbol{i}}$ is the $i$-th standard basis vector. We refer to a point in $\mathbb{R}_{\geqslant 0}^{n}$ as a $k$-diagonal point if it is any coordinate permutation of a point of the form $\boldsymbol{c}_{k}(d)$ for some $d>0$.

Lemma 3.1. If $1 \leqslant \ell \leqslant n-1,1<R<\infty$, and $\boldsymbol{z}$ is a maxima of $U(\boldsymbol{x})$ on $\Omega(R)$ then $\boldsymbol{z}$ is a $k$-diagonal point for some $k$ with $1 \leqslant k \leqslant n$.

We will prove Lemma 3.1 in Section 3.2 using the method of Lagrange multipliers (see Theorem 3.3).

By the symmetry of $f$ and $\eta_{\ell}$, if we restrict our attention to a single point $\boldsymbol{x} \in \mathbb{R}_{\geqslant 0}^{n}$, we may assume that there is some $0 \leqslant k \leqslant n$ such that $x_{1}, \ldots, x_{k}>0$ and $x_{k+1}=\cdots=x_{n}=$ 0 . With this in mind, we generalize the functions defined in (1.3) and (1.4) by setting

$$
\begin{aligned}
\eta_{\ell, k}(\boldsymbol{x}) & :=\eta_{\ell}\left(x_{1}, \ldots, x_{k}, 0, \ldots, 0\right), \\
\mu_{\ell, k}(y) & :=\eta_{\ell}(\underbrace{y, \ldots, y}_{k \text { times }}, 0, \ldots, 0) .
\end{aligned}
$$

These are related to our earlier definitions by $\eta_{\ell}=\eta_{\ell, n}$ and $\mu_{\ell}=\mu_{\ell, n}$, and we also have the further relations

$$
\eta_{\ell, k}(\boldsymbol{x})=\sum_{\substack{I \subseteq[k] \\
|I| \leqslant \ell}} x_{I}, \quad \mu_{\ell, k}(y)=\eta_{\ell, k}(y, \ldots, y)=\sum_{j=0}^{\ell}\left(\begin{array}{l}
k \\
j
\end{array}\right) x^{j}
$$

and finally, $\eta_{\ell, k}(\boldsymbol{x})=\eta_{k, k}(\boldsymbol{x})=\prod_{i=1}^{k}\left(1+x_{i}\right)$ if $\ell \geqslant k$.

Lemma 3.2. If $0 \leqslant \ell \leqslant n, 1 \leqslant k \leqslant n$, and $y \geqslant 0$, then

$$
\mu_{\ell, n}^{k}(y) \leqslant \mu_{\ell, k}^{n}(y) .
$$

The inequality is tight if and only if $\ell=0, \ell=n, y=0$ or $k=n$.

We will prove Lemmas 3.1 and 3.2 in Sections 3.2 and 3.3, respectively. We now show how they imply Theorem 1.3. 
Proof of Theorem 1.3. If $\ell=0$ or $\boldsymbol{x}=0$, then all terms in the two inequalities are equal to 1 . If $x_{1}=\cdots=x_{n}=y$ then all terms are identically equal to $\mu_{\ell}(y)$. If $\ell=n$, the left inequality is an identity and the right inequality is an application of the arithmeticgeometric mean inequality. In general, the right inequality follows from Proposition 1.1 part 1 and Proposition 2.1 part 2. So it suffices to prove the left inequality in the case where $\boldsymbol{x} \in \mathbb{R}_{\geqslant 0}^{n}, \boldsymbol{x} \neq 0$ and $1 \leqslant \ell \leqslant n-1$.

Since $\eta_{\ell}$ is strictly increasing in each variable, a non-zero $\boldsymbol{x}$ is contained in the surface $\Omega(R)$ with $R=R(\boldsymbol{x})=\eta_{\ell}(\boldsymbol{x})>\eta_{\ell}(0)=1$. Since $\eta_{\ell}(\boldsymbol{x}) \geqslant 1+x_{i}$ for all $1 \leqslant i \leqslant n, \Omega(R)$ is bounded. Since $\eta_{\ell}(\boldsymbol{x})$ is continuous, $\Omega(R)$ is also closed, and hence compact.

This means that there exists at least one point $\boldsymbol{z} \in \Omega(R)$ at which $f(\boldsymbol{x})$ takes its maximum value on $\Omega(R)$. Since $R>1, \boldsymbol{z} \neq 0$ and, by Lemma 3.1, $\boldsymbol{z}=\boldsymbol{c}_{k}(d)$, for some $1 \leqslant k \leqslant n$ and $d>0$. By Lemma 3.2, if $k<n$, then

$$
f(\boldsymbol{z})=\left(\mu_{\ell, n}(d)\right)^{\frac{k}{n}}<\mu_{\ell, k}(d)=\eta_{\ell}(\boldsymbol{z})=R .
$$

However, if $k=n$, we have $f(\boldsymbol{z})=\mu_{\ell}(d)=\eta_{\ell}(\boldsymbol{z})=R$.

\subsection{Lagrange multipliers and maxima}

In this section we prove Lemma 3.1 using Proposition 3.3.1 of [1], p.284. We restate this result as Theorem 3.3, a form more suitable to our purposes.

Theorem 3.3 (The method of Lagrange multipliers with inequality constraints.). Let $f, h_{1}, \ldots, h_{m}, g_{1}, \ldots, g_{r}: \mathbb{R}^{n} \rightarrow \mathbb{R}$ be continuously differentiable functions. Suppose $\boldsymbol{z}$ is a point at which $f(\boldsymbol{x})$ has a local maximum over $\Omega=\left\{\boldsymbol{x}: h_{1}(\boldsymbol{x})=\cdots=h_{m}(\boldsymbol{x})=\right.$ $\left.0, g_{1}(\boldsymbol{x}), \ldots, g_{r}(\boldsymbol{x}) \geqslant 0\right\}$. Suppose also that $\boldsymbol{z}$ is regular, i.e. $\left\{\nabla h_{1}(\boldsymbol{z}), \ldots, \nabla h_{m}(\boldsymbol{z})\right\} \cup$ $\left\{\nabla g_{i}(\boldsymbol{z}): i \in A(\boldsymbol{z})\right\}$ is a linearly independent set where $A(\boldsymbol{x}):=\left\{1 \leqslant j \leqslant r: g_{j}(\boldsymbol{x})=0\right\}$. Then there exist unique Lagrange multiplier vectors $\boldsymbol{\lambda}^{\prime} \in \mathbb{R}^{m}$ and $\boldsymbol{\rho}^{\prime} \in \mathbb{R}_{\geqslant 0}^{r}$, such that

$$
\begin{aligned}
\frac{\partial L}{\partial x_{i}}\left(\boldsymbol{z}, \boldsymbol{\lambda}^{\prime}, \boldsymbol{\rho}^{\prime}\right) & =0, \quad 1 \leqslant i \leqslant n, \\
\rho_{j}^{\prime} & =0, \quad j \notin A(z)
\end{aligned}
$$

where $L(\boldsymbol{x}, \boldsymbol{\lambda}, \boldsymbol{\rho}):=f(\boldsymbol{x})+\sum_{i=1}^{m} \lambda_{i} h_{i}(\boldsymbol{x})+\sum_{j=1}^{r} \rho_{j} g_{j}(\boldsymbol{x})$.

Proof of Lemma 3.1. Theorem 3.3 applies to the present setting with $\Omega(R)$ as the set $\Omega$, constraint function $h(\boldsymbol{x}):=\eta_{\ell}(\boldsymbol{x})-R$, and inequality constraints $g_{i}(\boldsymbol{x}):=x_{i}$ for $1 \leqslant i \leqslant n$. The Lagrangian function is then

$$
L(\boldsymbol{x}, \lambda, \boldsymbol{\rho}):=U(\boldsymbol{x})+\lambda\left(\eta_{l}(\boldsymbol{x})-R\right)+\sum_{i=1}^{n} \rho_{i} x_{i} .
$$

Let $\boldsymbol{z} \in \Omega(R)$ be a point at which $U(\boldsymbol{x})$ attains its maximum value over $\Omega(R)$. Since $R>1, \boldsymbol{z}$ has at least one non-zero coordinate. By symmetry we may assume $z_{1}, z_{2}, \ldots, z_{k}>0$ and $z_{k+1}=\ldots=z_{n}=0$ for some $k \geqslant 1$. Since $\nabla h(\boldsymbol{z})$ trivially has 
positive coordinates, $\nabla h(\boldsymbol{z})$ together with $\nabla g_{i}(\boldsymbol{z})=\boldsymbol{e}_{\boldsymbol{i}}$, for $k+1 \leqslant i \leqslant n$, form a linearly independent set. Thus $\boldsymbol{z}$ is regular, and the conditions of Theorem 3.3 are met.

The theorem statement now implies that there is a constant $\lambda^{\prime}$ such that

$$
\frac{1}{n} \frac{\mu_{\ell, n}^{\prime}\left(z_{i}\right)}{\mu_{\ell, n}\left(z_{i}\right)} U(\boldsymbol{z})+\lambda^{\prime} \frac{\partial \eta_{\ell}}{\partial x_{i}}(\boldsymbol{z})=0, \quad 1 \leqslant i \leqslant k .
$$

It is trivial to check that $U(\boldsymbol{z}) \geqslant 1$ and $\frac{\partial \eta_{\ell}}{\partial x_{i}}(\boldsymbol{z}) \geqslant 1$ for all $i$ with $1 \leqslant i \leqslant n$. Thus, if we define

$$
\gamma_{i}:=\frac{1}{n} \frac{\mu_{\ell, n}^{\prime}\left(z_{i}\right)}{\mu_{\ell, n}\left(z_{i}\right) \frac{\partial \eta_{\ell}}{\partial x_{i}}(\boldsymbol{z})}, \quad 1 \leqslant i \leqslant k,
$$

then $\gamma_{1}=\cdots=\gamma_{k}=-\lambda^{\prime} / f(z)$. Note that

$$
\gamma_{i}=\frac{\sum_{j=0}^{\ell-1}\left(\begin{array}{c}
n-1 \\
j
\end{array}\right) z_{i}^{j}}{\mu_{\ell, n}\left(z_{i}\right) \sum_{j=0}^{\ell-1} \sum_{\substack{I \subseteq[k]-i \\
|I|=j}} z_{I}}, \quad 1 \leqslant i \leqslant k .
$$

If $j, k$ are non-negative integers, define

$$
Z_{j, k}:=\sum_{\substack{I \subseteq[k] \backslash\{1,2\} \\|I|=j}} z_{I}
$$

Suppose that $z_{3}, \ldots, z_{k}$ are fixed. We will show that the equality $\gamma_{1}=\gamma_{2}$ holds if and only if $z_{1}=z_{2}$. By symmetry, this will then prove that $z_{1}=\cdots=z_{k}$, completing the proof that $\boldsymbol{z}$ is a $k$-diagonal point.

Observe that we can write

$$
\gamma_{1}=\frac{\sum_{j=0}^{\ell-1}\left(\begin{array}{c}
n-1 \\
j
\end{array}\right) z_{1}^{j}}{\mu_{\ell, n}\left(z_{1}\right)\left(\left(1+z_{2}\right) \sum_{j=0}^{\ell-2} Z_{j, k}+Z_{\ell-1, k}\right)},
$$

and

$$
\gamma_{2}=\frac{\sum_{j=0}^{\ell-1}\left(\begin{array}{c}
n-1 \\
j
\end{array}\right) z_{2}^{j}}{\mu_{\ell, n}\left(z_{2}\right)\left(\left(1+z_{1}\right) \sum_{j=0}^{\ell-2} Z_{j, k}+Z_{\ell-1, k}\right)} .
$$

Comparing (3.3) and (3.4), it is now clear that $\gamma_{1}=\gamma_{2}$ if and only if $\Gamma_{\ell, k}\left(z_{1}\right)=\Gamma_{\ell, k}\left(z_{2}\right)$, where

$$
\Gamma_{\ell, k}(y):=\frac{\sum_{j=0}^{\ell-1}\left(\begin{array}{c}
n-1 \\
j
\end{array}\right) y^{j} \cdot\left((1+y) \sum_{j=0}^{\ell-2} Z_{j, k}+Z_{\ell-1, k}\right)}{\mu_{\ell, n}(y)} .
$$


To conclude, we show that $\Gamma_{\ell, k}(y)$ is strictly decreasing on $[0, \infty)$ and, hence, one-toone. Note that

$$
\Gamma_{\ell, k}(y)=\widetilde{\Gamma}_{\ell, k}(y) \cdot\left(A+\frac{B}{1+y}\right),
$$

where

$$
\widetilde{\Gamma}_{\ell, k}(y):=\frac{\sum_{j=0}^{\ell-1}\left(\begin{array}{c}
n-1 \\
j
\end{array}\right) y^{j} \cdot(1+y)}{\mu_{\ell, n}(y)} .
$$

and where $A, B>0$ are constants. We show that $\widetilde{\Gamma}_{\ell, k}^{\prime}(y)<0$. This implies $\widetilde{\Gamma}_{\ell, k}(y)$ is strictly decreasing and hence $\Gamma_{\ell, k}(y)$ is strictly decreasing as well. Simple algebra shows that

$$
\widetilde{\Gamma}_{\ell, k}(y)=1-\frac{\left(\begin{array}{c}
n-1 \\
\ell
\end{array}\right) y^{\ell}}{\sum_{j=0}^{\ell}\left(\begin{array}{c}
n \\
j
\end{array}\right) y^{j}},
$$

and thus

$$
\widetilde{\Gamma}_{\ell, k}^{\prime}(y)=\left(\begin{array}{c}
n-1 \\
\ell
\end{array}\right) \cdot \frac{-\ell y^{\ell-1} \sum_{j=0}^{\ell}\left(\begin{array}{l}
n \\
j
\end{array}\right) y^{j}+y^{\ell} \sum_{j=0}^{\ell} j\left(\begin{array}{l}
n \\
j
\end{array}\right) y^{j-1}}{\mu_{\ell, n}(y)^{2}} .
$$

The numerator simplifies to

$$
-z^{\ell} \sum_{j=0}^{\ell}(\ell-j)\left(\begin{array}{c}
n \\
j
\end{array}\right) y^{j}<0,
$$

and the proof is complete.

\subsection{Majorization and polynomial inequalities}

In this section we use a partial order on polynomials in order to prove Lemma 3.2. Let $f(y)=\sum_{n \geqslant 0} c(n) y^{n}$ and $g(y)=\sum_{n \geqslant 0} d(n) y^{n}$ be polynomials in $y$ with real coefficients. We say that $f$ is dominated by $g$ in the coefficient partial order, denoted $f \sqsubseteq g$, if and only if $c(n) \leqslant d(n)$ for all $n \geqslant 0$. If $f \sqsubseteq g$, and $c(n)<d(n)$ for some $n \geqslant 0$, then we denote this by $f \sqsubset g$. We write $0 \sqsubseteq f$ if and only if $f$ has all coefficients non-negative and $0 \sqsubset f$ if and only $f$ has all coefficients non-negative and at least one coefficient positive.

Proposition 3.4. If $a>b \geqslant 0$ are integers, then

$$
\mu_{\ell, a}(y) \mu_{\ell, b}(x) \sqsubseteq \mu_{\ell, a-1}(x) \mu_{\ell, b+1}(x) .
$$

We have $\mu_{\ell, a}(x) \mu_{\ell, b}(x) \sqsubset \mu_{\ell, a-1}(x) \mu_{\ell, b+1}(x)$ if and only if, additionally, $a \geqslant b+2, a-1 \geqslant \ell$, and $\ell \geqslant 1$.

The proof of Proposition 3.4 requires the two following lemmas. 
Lemma 3.5. If $A, B, M, N$ are integers with $A \geqslant B \geqslant 0$ and $M \geqslant N \geqslant 0$, then

$$
\left(\begin{array}{c}
A \\
M
\end{array}\right)\left(\begin{array}{l}
B \\
N
\end{array}\right) \geqslant\left(\begin{array}{l}
A \\
N
\end{array}\right)\left(\begin{array}{l}
B \\
M
\end{array}\right)
$$

For these same ranges of parameters, the inequality is strict if and only if $A>B, M>N$, $A \geqslant M$, and $B \geqslant N$.

Proof. If $A=B$ or $M=N$ then both sides of the inequality are identically equal. If $M>A$, then $M>B$ and both sides are 0 . If $N>B$, then $M>B$ and, again, both sides are 0 . We may now assume $A>B, M>N, A \geqslant M$, and $B \geqslant N$. Canceling factorials, the desired inequality becomes

$$
(A-N)_{M-N}>(B-N)_{M-N}
$$

where, for non-negative integers $n$ and real numbers $\alpha,(\alpha)_{n}:=\prod_{i=0}^{n-1}(x-i)$ is the falling factorial. Since $(\alpha)_{n}>(\beta)_{n}$ if $\alpha>\beta \geqslant n-1 \geqslant 0$, we are done.

Lemma 3.6. Suppose that $f_{1}, f_{2}, g_{1}, g_{2}$ are polynomials. If $0 \sqsubseteq f_{1} \sqsubseteq f_{2}$ and $0 \sqsubseteq g_{1} \sqsubseteq g_{2}$, then $f_{1} g_{1} \sqsubseteq f_{2} g_{2}$. If, additionally, $f_{1} \sqsubset f_{2}$ and $0 \sqsubset g_{2}$, then $f_{1} g_{1} \sqsubset f_{2} g_{2}$.

Proof. Denoting the coefficients by $f_{i}=\sum_{j \geqslant 0} f_{i, j} y^{j}$ and $g_{i}=\sum_{j \geqslant 0} g_{i, j} y^{j}$ for $i=1$, 2, the conditions of the lemma state that $0 \leqslant f_{1, j} \leqslant f_{2, j}$ and $0 \leqslant g_{1, j} \leqslant g_{2, j}$ for all $j \geqslant 0$. Writing their products as $f_{1} g_{1}=\sum_{l \geqslant 0} a_{l} y^{l}$ and $f_{2} g_{2}=\sum_{l \geqslant 0} b_{l} y^{l}$, the coefficients then satisfy

$$
a_{l}=\sum_{j, k \geqslant 0, j+k=l} f_{1, j} g_{1, k} \leqslant \sum_{j, k \geqslant 0, j+k=l} f_{2, j} g_{2, k}=b_{l},
$$

since $f_{1, j} g_{1, k} \leqslant f_{2, j} g_{2, k}$ for all $j, k \geqslant 0$.

If there is additionally some pair $j, k$ such that $0 \leqslant f_{1, j}<f_{2, j}$ and $0<g_{2, k}$, then $f_{1, j} g_{1, k}<f_{2, j} g_{2, k}$, and therefore the stronger conclusion $a_{j+k}<b_{j+k}$ holds.

Proof of Proposition 3.4. By definition, proving that $\mu_{\ell, a}(y) \mu_{\ell, b}(y) \sqsubseteq \mu_{\ell, a-1}(y) \mu_{\ell, b+1}(y)$ is the same as proving that for each $0 \leqslant m \leqslant 2 \ell$ we have

$$
\sum_{d=M^{\prime}}^{M}\left(\begin{array}{l}
a \\
d
\end{array}\right)\left(\begin{array}{c}
b \\
m-d
\end{array}\right) \leqslant \sum_{d=M^{\prime}}^{M}\left(\begin{array}{c}
a-1 \\
d
\end{array}\right)\left(\begin{array}{c}
b+1 \\
m-d
\end{array}\right)
$$

where $M^{\prime}:=\max \{0, m-\ell\}$ and $M:=\min \{\ell, m\}$. Likewise, the stronger condition that $\mu_{\ell, a}(y) \mu_{\ell, b}(y) \sqsubset \mu_{\ell, a-1}(y) \mu_{\ell, b+1}(y)$ is equivalent to additionally proving that there is an $m$ with $0 \leqslant m \leqslant 2 \ell$ for which (3.6) is strict. Applying Pascal's identity $\left(\begin{array}{l}a \\ d\end{array}\right)=\left(\begin{array}{c}a-1 \\ d\end{array}\right)+\left(\begin{array}{c}a-1 \\ d-1\end{array}\right)$ to the left-side and $\left(\begin{array}{c}b+1 \\ m-d\end{array}\right)=\left(\begin{array}{c}b \\ m-d\end{array}\right)+\left(\begin{array}{c}b \\ m-d-1\end{array}\right)$ to the right, and then cancelling like terms, (3.6) becomes

$$
\sum_{d=M^{\prime}}^{M}\left(\begin{array}{l}
a-1 \\
d-1
\end{array}\right)\left(\begin{array}{c}
b \\
m-d
\end{array}\right) \leqslant \sum_{d=M^{\prime}}^{M}\left(\begin{array}{c}
a-1 \\
d
\end{array}\right)\left(\begin{array}{c}
b \\
m-d-1
\end{array}\right)
$$


Furthermore, after a summation index shift all of the terms but one in (3.7) cancel, leaving only $d=M^{\prime}$ on the left-side and $d=M$ on the right:

$$
\left(\begin{array}{c}
a-1 \\
M^{\prime}-1
\end{array}\right)\left(\begin{array}{c}
b \\
m-M^{\prime}
\end{array}\right) \leqslant\left(\begin{array}{c}
a-1 \\
M
\end{array}\right)\left(\begin{array}{c}
b \\
m-M-1
\end{array}\right) .
$$

If $M^{\prime}=0$, then this inequality is trivially satisfied, and thus so is (3.6). We therefore need only consider the case that $M^{\prime}=m-\ell$. This means that $m \geqslant \ell$, so in this case $M=\ell$, and (3.6) is finally equivalent to the inequality

$$
\left(\begin{array}{c}
a-1 \\
m-\ell-1
\end{array}\right)\left(\begin{array}{l}
b \\
\ell
\end{array}\right) \leqslant\left(\begin{array}{c}
a-1 \\
\ell
\end{array}\right)\left(\begin{array}{c}
b \\
m-\ell-1
\end{array}\right) .
$$

We now apply Lemma 3.5 with $A=a-1, B=b, M=\ell, N=m-\ell-1$ to complete the proof. The inequality easily follows. It is also easy to see that (3.6) is strict in the cases where $a \geqslant b+2, a-1 \geqslant \ell$, and $2 \ell \geqslant m \geqslant \ell+1$ (and hence $\ell \geqslant 1$ ).

Remark 3.7. Proposition 3.4 (and its proof) can also be interpreted combinatorially. In particular, consider two rows consisting of $a$ and $b$ square cells, respectively. The $y^{m}$ coefficient in

$$
\sum_{i=0}^{\ell}\left(\begin{array}{l}
a \\
i
\end{array}\right) y^{i} \cdot \sum_{j=0}^{\ell}\left(\begin{array}{l}
b \\
j
\end{array}\right) y^{j}
$$

is the number of ways of marking exactly $m$ of the cells subject to the restriction that there are at most $\ell$ marked cells in each row, and the result then states that if $a>b$, then there are at least as many ways to mark two rows of length $a-1$ and $b+1$ subject to the same restriction.

Corollary 3.8. If $y \geqslant 0, \boldsymbol{\lambda}_{\mathbf{1}}, \boldsymbol{\lambda}_{\mathbf{2}} \in \Delta(m)$ each have integer coordinates, and $\boldsymbol{\lambda}_{\mathbf{1}} \succeq \boldsymbol{\lambda}_{\mathbf{2}}$ then

$$
\prod_{i=1}^{m} \mu_{\ell, \lambda_{1}(i)}(y) \sqsubseteq \prod_{i=1}^{m} \mu_{\ell, \lambda_{2}(i)}(y) .
$$

Proof. Suppose $\boldsymbol{\lambda}_{\mathbf{1}} \neq \boldsymbol{\lambda}_{\mathbf{2}}$. By definition, there must then be two indices $1 \leqslant \alpha<\beta \leqslant m$ such that $\lambda_{1}(\alpha)>\lambda_{2}(\alpha)$ and $\lambda_{1}(\beta)<\lambda_{2}(\beta)$. Define $\boldsymbol{\lambda}_{1}^{\prime}$ by setting

$$
\lambda_{1}^{\prime}(\alpha):=\lambda_{1}(\alpha)-1, \quad \lambda_{1}^{\prime}(\beta):=\lambda_{1}(\beta)+1,
$$

and $\lambda_{1}^{\prime}(i):=\lambda_{1}(i)$ for all $i \neq \alpha, \beta$. Importantly, it is still true that $\boldsymbol{\lambda}_{\mathbf{1}}^{\prime}$ majorizes $\boldsymbol{\lambda}_{\mathbf{2}}$.

Noting that $\lambda_{1}(\alpha)>\lambda_{2}(\alpha) \geqslant \lambda_{2}(\beta)>\lambda_{1}(\beta)$, Proposition 3.4 now states that

$$
\mu_{\ell, \lambda_{1}(\alpha)}(y) \mu_{\ell, \lambda_{1}(\beta)}(y) \sqsubseteq \mu_{\ell, \lambda_{1}^{\prime}(\alpha)}(y) \mu_{\ell, \lambda_{1}^{\prime}(\beta)}(y)
$$

which, combined with Lemma 3.6, implies that

$$
\prod_{i=1}^{m} \mu_{\ell, \lambda_{1}(i)}(x) \sqsubseteq \prod_{i=1}^{m} \mu_{\ell, \lambda_{1}^{\prime}(i)}(x) .
$$


If $\boldsymbol{\lambda}_{\mathbf{1}}^{\prime}=\boldsymbol{\lambda}_{\mathbf{2}}$, then (3.8) gives the statement of the corollary. Otherwise, the above procedure is repeated (a finite number of steps) until this is the case.

Applying this result with the partitions $\boldsymbol{\lambda}_{\mathbf{1}}=n^{k}$ and $\boldsymbol{\lambda}_{\mathbf{2}}=k^{n}$ will finally complete the proof of Lemma 3.2.

Proof of Lemma 3.2. Corollary 3.8 implies $\mu_{\ell, n}(y)^{k} \sqsubseteq \mu_{\ell, k}(y)^{n}$. Since this partial order requires that all coefficients be dominated, this immediately implies that $\mu_{\ell, n}(y)^{k} \leqslant \mu_{\ell, k}(y)^{n}$ for all $y \geqslant 0$. Clearly, if $k=n, \ell=0, \ell=n$, or $x=0$, then $\mu_{\ell, n}(x)^{k}=\mu_{\ell, k}(x)^{n}$. It therefore remains to be shown that the inequality is strict if $1 \leqslant \ell \leqslant n-1, k \leqslant n-1$ and $x>0$.

Proposition 3.4 implies that $\mu_{\ell, n} \mu_{\ell, 0} \sqsubset \mu_{\ell, n-1} \mu_{\ell, 1}$. Following the proof method of Proposition 3.4, we introduce the dummy term $\mu_{\ell, 0}=1$ and find

$$
\mu_{\ell, n}^{k}=\mu_{\ell, n}^{k-1}\left(\mu_{\ell, n} \mu_{\ell, 0}\right) \sqsubset \mu_{\ell, n}^{k-1}\left(\mu_{\ell, n-1} \mu_{\ell, 1}\right) \sqsubseteq \mu_{\ell, k}^{n} .
$$

The second relation follows from Lemma 3.6, and the third follows from Corollary 3.8. Since $\mu_{\ell, n}^{k} \sqsubset \mu_{\ell, k}^{n}$ and $x>0$, we conclude that $\mu_{\ell, n}(x)^{k}<\mu_{\ell, k}(x)^{n}$

\section{Inequalities for sums of Bernoulli random variables}

In this brief section we describe the relationship between our quasi-mean inequalities in Theorem 1.3 and the distributions of sums of Bernoulli random variables.

Proof of Theorem 1.4. The inequality is trivial if $\ell=n$, so we henceforth assume that $\ell<n$. Furthermore, if $p_{i}=1$ for some $i$ with $1 \leqslant i \leqslant n$, then $\mathbf{P}\left(C_{i} \leqslant \ell\right)=0$ and the inequality is again trivially true. We therefore also assume that $p_{i} \in[0,1)$ for each $i$.

All of the events $\left\{C_{j} \leqslant \ell\right\}$ are independent, and their individual probabilities are given by

$$
\mathbf{P}\left(C_{j} \leqslant \ell\right)=\sum_{0 \leqslant m \leqslant \ell}\left(\begin{array}{c}
n \\
m
\end{array}\right) p_{j}^{m}\left(1-p_{j}\right)^{n-m} .
$$

Thus

$$
\mathbf{P}\left(\max _{1 \leqslant j \leqslant n}\left\{C_{j}\right\} \leqslant \ell\right)=\prod_{j=1}^{n} \sum_{0 \leqslant m \leqslant \ell}\left(\begin{array}{c}
n \\
m
\end{array}\right) p_{j}^{m}\left(1-p_{j}\right)^{n-m} .
$$

Similarly, the events $\left\{R_{i} \leqslant \ell\right\}$ are also independent, and their probabilities are given by

$$
\mathbf{P}\left(R_{i} \leqslant \ell\right)=\sum_{0 \leqslant m \leqslant \ell} \sum_{\substack{I \subseteq[n] \\|I|=m}} \prod_{j \in I} p_{j} \prod_{j \notin I}\left(1-p_{j}\right),
$$

SO

$$
\mathbf{P}\left(\max _{1 \leqslant i \leqslant n}\left\{R_{i}\right\} \leqslant \ell\right)=\left(\sum_{0 \leqslant m \leqslant \ell} \sum_{\substack{I \subseteq[n] \\|I|=m}} \prod_{j \in I} p_{j} \prod_{j \notin I}\left(1-p_{j}\right)\right)^{n} .
$$

THE ELECTRONiC JOURNAL OF COMBINATORics 20(3) (2013), \#P34 
Dividing (4.1) and (4.2) by $\prod_{j=1}^{n}\left(1-p_{j}\right)$, we see that the desired inequality is equivalent to the left inequality from Theorem 1.3 with $x_{i}=p_{i} /\left(1-p_{i}\right)$ for $1 \leqslant i \leqslant n$.

\section{References}

[1] D. Bertsekas, Nonlinear Programming, Athena Scientific, Belmont, MA, 1995.

[2] G. Hardy, J. Littlewood, and G. Pólya, Inequalities, 2nd. ed., Cambridge University Press, Cambridge, 1952.

[3] R. Muirhead, Some methods applicable to identities of symmetric algebraic functions of $n$ letters, Proc. Edinburgh Math. Soc. 21 (1902/03), 144-157.

[4] R. Stanley, Enumerative Combinatorics, vol. 2, Cambridge University Press, Cambridge, 1999. 